\title{
MISE EN ÉVIDENCE DES DÉPLACEMENTS D'ANGUILLES SÉDENTAIRES (ANGUILLA ANGUILLA L.) EN RELATION AVEC LE CYCLE LUNAIRE DANS LE LAC DE GRAND-LIEU (LOIRE-ATLANTIQUE).
}

\author{
G. ADAM $(1,2)$ et P. ELIE (1)
}

(1) CEMAGREF, Groupement de Bordeaux, Division Aquaculture et Pêche, 50 avenue de Verdun, B.P. 3, 33611 GAZINET Cedex, France.

(2) Université Paul Sabatier, Laboratoire d'hydrobiologie, 118 route de Narbonne, 31062 TOULOUSE Cedex, France.

\section{RÉSUMÉ}

Nous cherchons par ce travail à mettre en évidence l'influence du cycle lunaire sur les déplacements d'anguilles européennes sédentaires (Anguilla anguilla L. 1758). L'évolution des captures par engin de pêche passif révèle les déplacements de l'anguille. Un système de carnet de pêche journalier a été mis en place en relation avec la pêcherie professionnelle du lac de Grand-Lieu (Loire-Atlantique). Il permet de collecter des données fines de capture. Cet article présente l'analyse des fluctuations journalières des prises par unité d'effort (CPUE) d'anguille européenne sur une période de deux ans (1991 et 1992). Deux éléments ressortent de cette étude. Tout d'abord, la série chronologique des CPUE suit une tendance annuelle qui la conduit de valeurs hivernales plutôt faibles aux valeurs estivales et automnales fortes. Mais à l'intérieur de cette tendance annuelle des variations plus courtes se dessinent. La recherche d'un événement cyclique par la transformée de FOURIER n'a donné que des résultats imprécis. Mais un filtrage "passe-bas" des données a, par contre, permis de confirmer l'hypothèse émise sur l'existence d'une périodicité d'événements autour de 28 jours. Cette périodicité correspond parfaitement au cycle lunaire. Enfin, il est proposé d'affiner l'étude par l'analyse des relations entre les CPUE et les données environnementales de type météorologique.

\section{MOVEMENTS OF THE SEDENTARIES EELS (ANGUILLA ANGUILLA L.) REVEALED IN RELATION TO LUNAR CYCLE INTO THE LAKE OF GRAND-LIEU (LOIRE-ATLANTIQUE).}

\section{SUMMARY}

The aim of this work is to study the influence of lunar cycle on movements of sedentaries European eels (Anguilla anguilla L. 1758). The evolution of catches of passive fishing gears reveal movements of eels. A daily logbook system had been settled in relation with professional fishery of the lake of Grand-Lieu (Loire-Atlantique). It allows to collect accurate catch data. This paper presents an analysis of daily fluctuations of catch per unit effort (CPUE) of European eel during two years (1991 and 1992). Two elements are result of this study. First, the time serie of CPUE follows an annual trend which leads from low spring values to highest summer and autumnal values. But into this annual trend, shortless variations exist. Second, research of cyclic occurrence by the FOURIER transform analysis has produced only imprecise results. But a lowpass filter, on the other hand, confirms the hypothesis of the presence of a 28 days frequency. This periodicity perfectly corresponds to lunar cycle. Finally, it is proposed to improve the study with a CPUE and meteorological datas relations analysis. 


\section{INTRODUCTION}

Le comportement de l'anguille a déjà fait l'objet de nombreux travaux, chaque auteur s'efforçant de lier le comportement aux facteurs de l'environnement. Mais un examen de la littérature montre que $56 \%$ des publications traitent de la phase de migration anadrome de l'anguille (cf. ELIE et ROCHARD, 1994), un autre tiers s'intéresse à la phase de dévalaison des anguilles argentées subadultes (en particulier : BOETIUS, 1967 ; TESCH, 1977 ; TODD, 1981 ; HARALDSTAD et al., 1985 ; VOLLESTAD et al., 1986). Finalement, $10 \%$ seulement se penche sur la période de sédentarisation de l'anguille en eau continentale.

Or, les civelles en phase de colonisation des eaux continentales ou les anguilles d'avalaison cherchant à rejoindre leur site de reproduction ne répondent pas aux mêmes préoccupations que les anguilles sédentaires. Le comportement de ces dernières, en phase de croissance, repose essentiellement sur la recherche d'un territoire vital et la recherche de nourriture.

Le déplacement des anguilles peut être observé au moyen d'engins de pêche de type passif. Une étude piscicole et halieutique du lac de Grand-Lieu (Loire-Atlantique), réalisée entre 1990 et 1992 (ADAM et ELIE, 1993), nous a conduits à collecter des données journalières de capture. Obtenues auprès de la pêcherie professionnelle, les données de production précises sont examinées en terme de «Biomasse capturée par unité d'effort".

Si de nombreux auteurs ont montré l'influence du cycle lunaire dans les déplacements de l'anguille d'avalaison (17 auteurs au moins), beaucoup moins se sont préoccupés de ce thème vis-à-vis des civelles (DEELDER, 1960 et 1970 ; CREUTZBERG, 1961 ; FINIGER, 1976 ; ELIE, 1979 ; DAHL, 1983 ; JELLYMAN et RYAN, 1983). Parmi ces cinq auteurs, DAHL (1983) a mis en évidence l'influence de la lune sur les anguillettes juste pigmentées, encore en phase de migration et sous influence de la marée dynamique. Les auteurs se préoccupent souvent de phénomènes associés au cycle lunaire tels que la marée (cf. ELIE et ROCHARD, 1994). Mais, seuls WESTIN et NYMAN (1979) ont étudié cette influence sur l'anguille jaune sédentaire. Toutefois ces auteurs précisent d'une part que la température est un facteur prédominant pour expliquer les déplacements de l'anguille, et d'autre part que la liaison entre activité de l'anguille jaune et le cycle lunaire est plus faible que pour l'anguille d'avalaison.

Notre objectif est ici de suivre l'évolution des déplacements d'anguille européenne (Anguilla anguilla $L$. 1758) révélée par l'évolution des captures par engins de pêche passifs, de type capetchades, et de chercher l'influence éventuelle d'un rythme lunaire sur cette évolution. En effet, l'existence d'un cycle dans l'évolution des captures est souvent envisagée par expérience ; le cycle lunaire de période 28 jours environ est, par exemple, évoqué par les pêcheurs professionnels d'anguille pour rappeler les baisses de prises enregistrées les nuits de pleine lune.

\section{ZONE D'ETUDE ET METHODOLOGIE}

\section{Description du lac et de la pêcherie}

\subsection{Le lac de Grand-Lieu}

Le lac de Grand-Lieu se situe à 14 kilomètres au sud-ouest de Nantes dans le département de Loire-Atlantique. II s'étend entre $1^{\circ} 38^{\prime}$ et $1^{\circ} 45^{\prime}$ de longitude Ouest et entre $47^{\circ} 02^{\prime}$ et $47^{\circ} 08^{\prime}$ de latitude Nord.

Ses principaux affluents sont La Boulogne et L'Ognon ayant des longueurs respectives de 100 et 30 kilomètres. Les eaux du lac s'écoulent en aval, vers le nord-ouest, dans un émissaire unique : l'Acheneau conduisant à l'estuaire de la Loire (Figure 1). Deux systèmes de vannages permettent de contrôler les eaux du système Loire-AcheneauGrand-Lieu. Pour permettre à l'anguille le franchissement des barrages au cours de sa phase de migration anadrome, des passes à civelle ont été mises en place en 1992. 

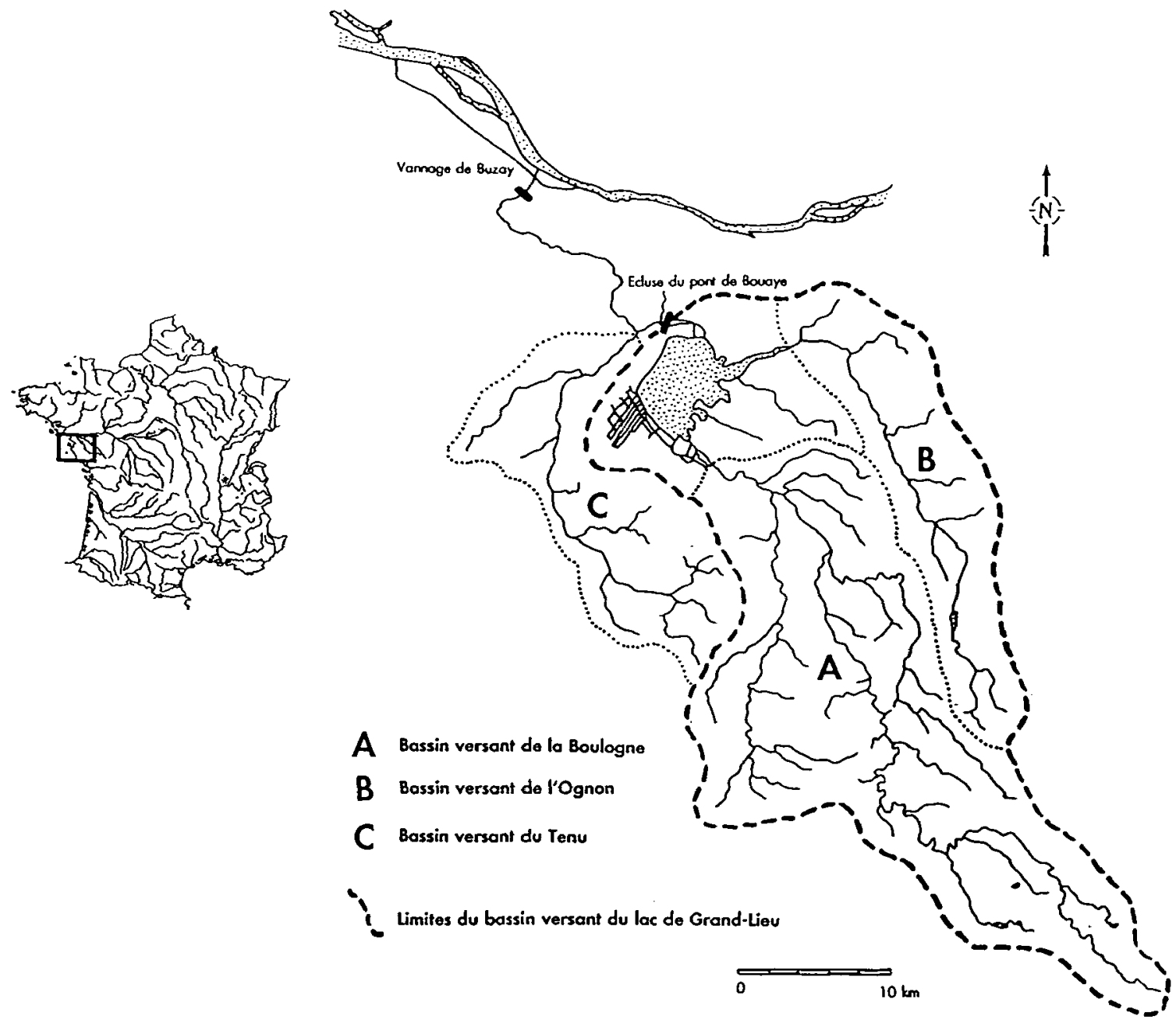

Figure 1 : Réseau hydrographique et bassin versant du lac de Grand-Lieu.

Figure 1 : Water bodies and drainage basin of lake of Grand-Lieu (Loire-Atlantique).

La faible profondeur du lac et le relief de ses fonds peu marqués conduisent à de très grandes variations des surfaces inondées pour des variations minimes du niveau des eaux. Sa surface varie ainsi de 3900 à 6300 hectares de l'été à l'hiver, saison au cours de laquelle le lac de Grand-Lieu se place au premier rang d'importance des lacs de plaine français.

\subsection{La pêcherie professionnelle}

La pratique de la pêche y est très ancienne. Les pêcheurs professionnels du lac de Grand-Lieu sont regroupés en société coopérative depuis 1907. Les effectifs des pêcheurs n'ont cessé de décroitre depuis le début du siècle. De 120 en 1920, le nombre de pêcheurs se réduit aujourd'hui aux 15 membres de la Société coopérative des pêcheurs de GrandLieu. Huit pêcheurs travaillent à temps plein et utilisent la totalité des quotas des engins fixés par les réglementations, deux autres travaillent à mi-temps.

Parmi les engins de pêche utilisés pour capturer l'anguille dans le lac de Grand-Lieu, le verveux à trois ailes est de loin le plus utilisé. Plus communément appelé verveux, cet engin est l'équivalent de la capetchade méditerranéenne. II fut introduit à Grand-Lieu dans les années 1970 par un pêcheur et est devenu très vite l'engin principal utilisé dans la pêcherie. Chaque pêcheur est limité à n'utiliser que 10 verveux constitués de filet à mailles 
carrées de 10 millimètres de côté. Le verveux est un engin passif constitué d'une paradière (généralement de 100 mètres de long), conduisant le poisson vers un enclos triangulaire dont les angles se terminent en pièges (poches). Les pièges sont aussi en filets souples maintenus en formes cylindriques par des arceaux en plastique. Le maillage des différents filets composant les verveux varient, la paradière et l'enclos triangulaire sont équipés d'un maillage plus important (14 $\mathrm{mm}$ de côté de maille) que le piège final (10 $\mathrm{mm}$ de côté de maille). Les verveux sont placés dans des zones comprises entre 60 et $150 \mathrm{~cm}$ de profondeur c'est-à-dire pratiquement sur l'ensemble des parties en eau du lac.

\section{Collecte des données de capture}

Les pêcheurs professionnels du lac de Grand-Lieu sont tenus de rendre compte de leurs captures à la préfecture du département de Loire-Atlantique par des déclarations mensuelles. Ces données renseignent déjà sur les prises de la pêcherie, mais l'unité de mesure n'est pas assez précise pour permettre une analyse fine de l'évolution des captures et la mise en relation avec certains phénomènes biologiques. De surcroît, les déclarations ne renseignent pas sur l'effort de pêche déployé par la pêcherie, le manque d'informations dans ce domaine nous a obligés à envisager un autre système de collecte des données de captures plus précis et plus complet.

Pour pouvoir estimer les captures par unité d'effort (CPUE = captures par engin sur un site et pour un jour de pêche), l'instauration du carnet de pêche journalier s'est révélée indispensable. Chaque pêcheur doit remplir une page de son carnet mensuel par jour de pêche et par type d'engin utilisé. La mise au point du carnet de pêche journalier a débuté dès septembre 1990 ; depuis cette date et sans discontinuer, la tenue du carnet se poursuit. Neuf pêcheurs sur dix ont décidé, dès le début de cette opération, de participer activement à ce recueil d'informations. Le suivi de cette pêcherie est pour nous un formidable moyen d'échantillonnage avec un engin standard.

Pour notre analyse des CPUE d'anguille, nous n'utiliserons que quelques rubriques du carnet : le type et le nombre d'engins utilisés, le nombre de jours de pêche des engins, et le poids estimé ou mesuré d'anguilles capturées.

\section{Méthodes d'analyse de la série chronologique des CPUE d'anguille}

Nous posons l'hypothèse de l'existence d'un lien entre l'évolution des captures d'anguilles et le calendrier lunaire. Si ce lien existe, la série chronologique des captures aura nécessairement une évolution cyclique dont une fréquence remarquable aura pour valeur celle du cycle lunaire : $1 / 28$ jours $^{-1}$.

\subsection{Mise en évidence des fréquences remarquables}

A partir de notre série chronologique de CPUE d'anguille, nous cherchons à mettre en évidence l'existence de phénomènes périodiques. Pour cela, nous utilisons une méthode mathématique appelée : “Transformée de FOURIER». Cette méthode est habituellement utilisée pour dégager des fréquences "remarquables", si elles existent, à partir d'un signal bruité (GRAMAGLIA, 1993). Sans entrer plus en avant dans la description de la transformée de FOURIER, rappelons que cette technique nous aidera à chercher des fréquences «remarquables» en passant de l'espace temps à celui des fréquences.

\subsection{Filtrage de la série}

Pour éliminer de notre série les moyennes fréquences rendant difficile l'observation des tendances mensuelles, il est possible d'appliquer un filtre. Parmi le cortège des techniques, nous avons choisi un filtre d'affaiblissement qui ne laisse passer que les fréquences en dessous d'une valeur limite. C'est-à-dire, dans notre cas, que le filtre laissera passer les fréquences se rapportant à une période supérieure ou égale à 28 jours. Le filtre de "TCHEBYCHEV" est un de ces filtres dit passe-bas (BILDSTEIN, 1990). En d'autres termes, nous éliminons de nos données toutes évolutions basées sur une fréquence différente de $1 / 28$. 


\section{RÉSULTATS}

\section{Recherche de phénomènes cycliques}

La dynamique des captures d'anguilles par verveux répond à un signal naturellement bruité (Figure 2). De ce phénomène, il est possible d'entrevoir des grandes tendances d'évolution, mais elles sont masquées par des variations de hautes fréquences.

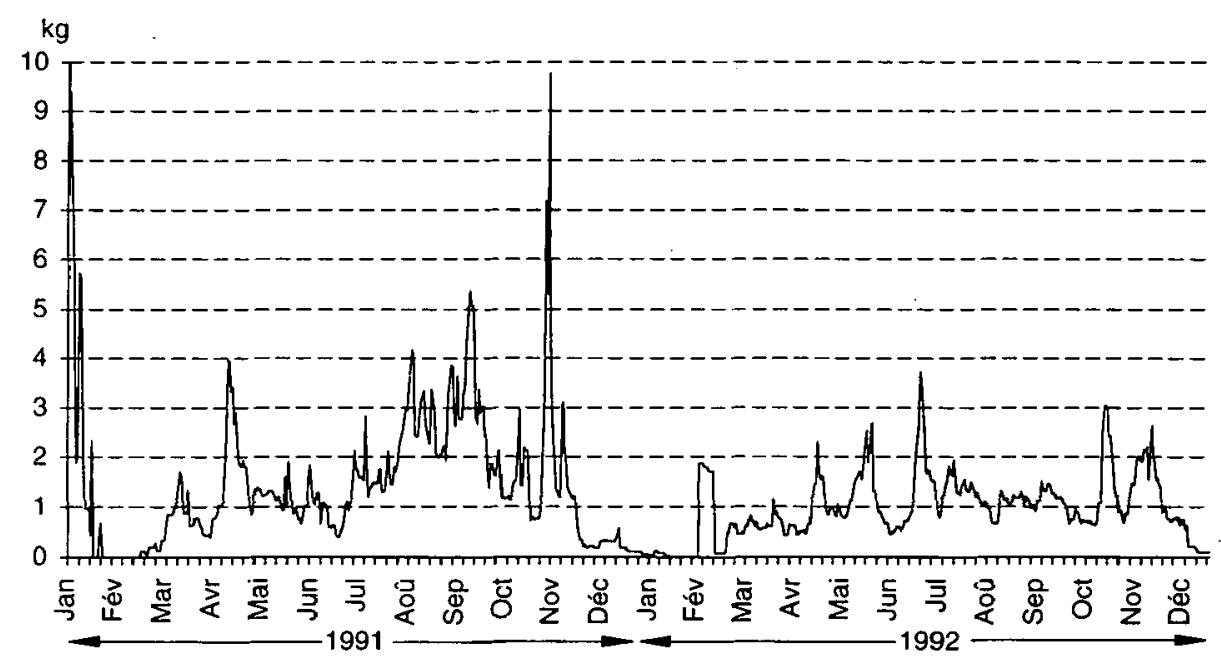

Figure 2 : Evolution journalière des captures d'anguille (Anguilla anguilla L.) par unité d'effort en 1991 et 1992 (Lac de Grand-Lieu, Loire-Atlantique).

Figure 2 : Daily evolution of catch per unit effort for eel (Anguilla anguilla L.) in 1991 and 1992 (Lake of Grand-Lieu, Loire-Atlantique).

Une première analyse a été réalisée sur l'ensemble des données de captures par unité d'effort entre le $1^{\text {er }}$ janvier 1991 et le 31 décembre 1992. L'observation de la transformée de FOURIER ne permet pas de mettre en évidence un phénomène fréquentiel bien net. Le phénomène apparaît plutôt bruité, de telle sorte qu'aucun pic de fréquence ne se détache franchement (Figure 3).

Mais nous avons pu remarquer que plusieurs grandes périodes saisonnières se découpent en 1991 comme en 1992. Ainsi, il est possible de séparer une saison printanière comprise entre le $1^{\text {er }}$ mars et le 30 juin, et une période plutôt estivale et automnale, comprise entre le $1^{\text {er }}$ juillet et le 22 octobre. De cette manière, nous simplifions en quelque sorte le problème, d'une part en éliminant les données concernant l'avalaison des anguilles, souvent considérées comme un phénomène particulier, d'autre part en isolant des périodes d'apparence plus homogènes.

Les transformées de FOURIER obtenues sur chacune de ces périodes ne révèlent pas non plus de fréquences majeures, mais une lecture attentive conduit tout de même à noter un pic remarquable de basse fréquence (Figures 3 ). Ce pic évolue autour des fréquences 0,0313 pour l'année 1991, 0,0341 pour le printemps 1992 et 0,0367 pour l'automne 1992. Cela correspond à peu près à des périodes respectives de 32,29 et 27 jours. 

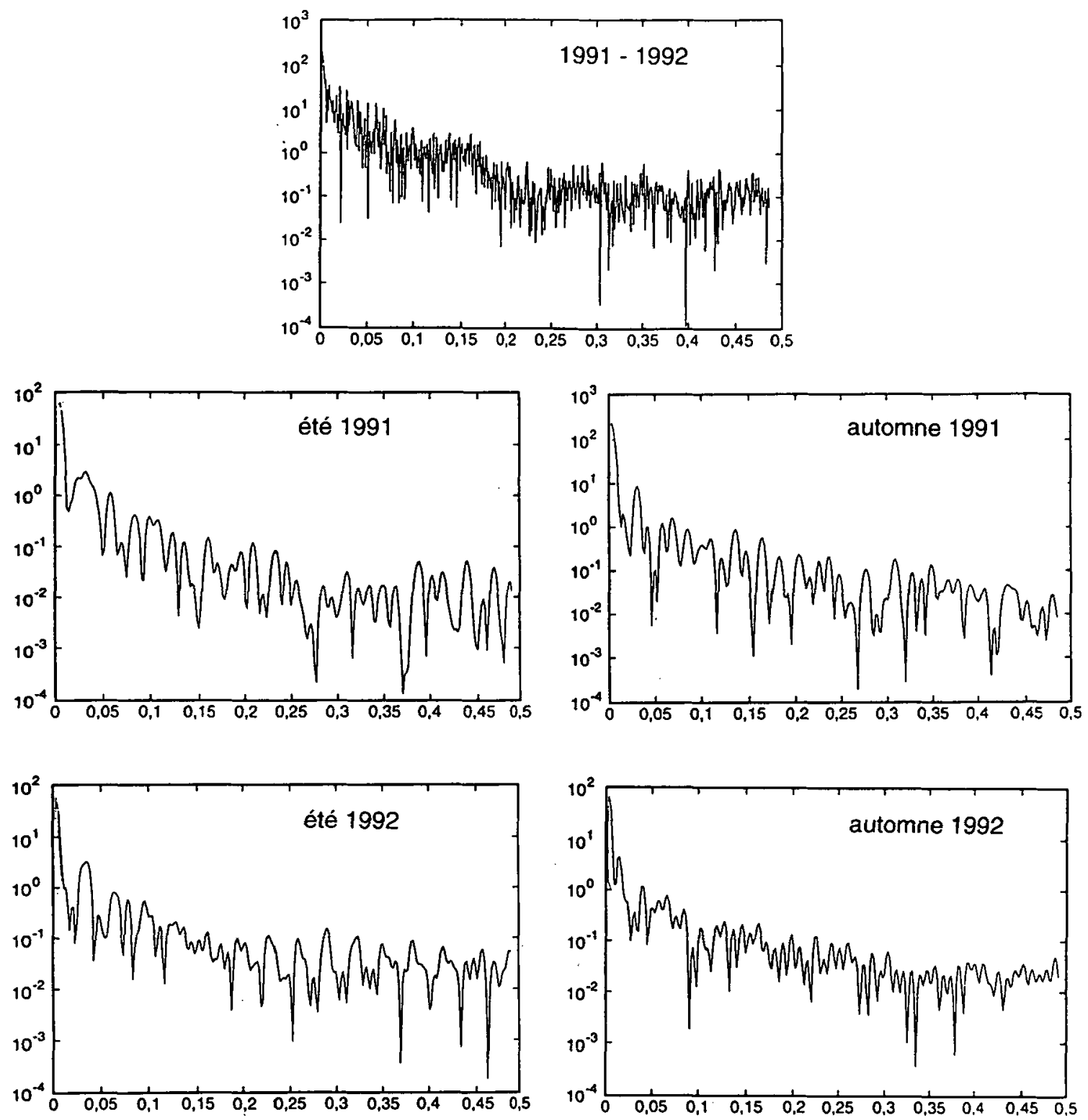

Figure 3 : Transformées de FOURIER sur les données de captures d'anguille (Anguilla anguilla L.) par unité d'effort (Lac de Grand-Lieu, LoireAtlantique). En abscisses, les fréquences (jours ${ }^{-1}$ ).

Figure 3 : FOURIER transform analysis on catch per unit effort for eel (Anguilla anguilla L.) (Lake of Grand-Lieu, Loire-Atlantique). Abscissa, Frequency (days $^{-1}$ ).

La précision de cette analyse ne permet pas de conserver la valeur exacte de la fréquence, mais plutôt de situer une zone dans laquelle la fréquence doit se trouver. La fréquence $1 / 28$ est intéressante : elle correspond à une réponse sur les transformées de FOURIER, elle est également connue pour être liée à de nombreux phénomènes lunaires. II est donc utile d'étudier l'évolution des captures à partir d'une observation focalisée dans une fenêtre d'observation de période de 28 jours. 


\section{Filtrage de la série sur 28 jours}

Notre série chronologique des captures journalières d'anguilles au verveux est, comme le montre la méthode des “transformées de FOURIER", extrêmement bruitée. II faut donc pouvoir éliminer ce bruit par une technique de filtrage.

Appliqué à nos données, le filtre bas de TCHEBYCHEV fournit une série lissée (Figure 4). La courbe restitue assez fidèlement l'évolution générale observée sur les données brutes. Une régression simple réalisée sur la base des données brutes et lissées donne pour résultat un coefficient de corrélation de 0,875 , ce qui montre que le filtrage ne déforme pas beaucoup la courbe originelle. Mais surtout, l'oscillation obtenue, autant sur l'année 1991 que sur l'année 1992, laisse à penser qu'un phénomène périodique de 28 jours module bien l'évolution des captures. Le cycle lunaire de période voisine de 28 jours peut être à l'origine de l'allure de la courbe.
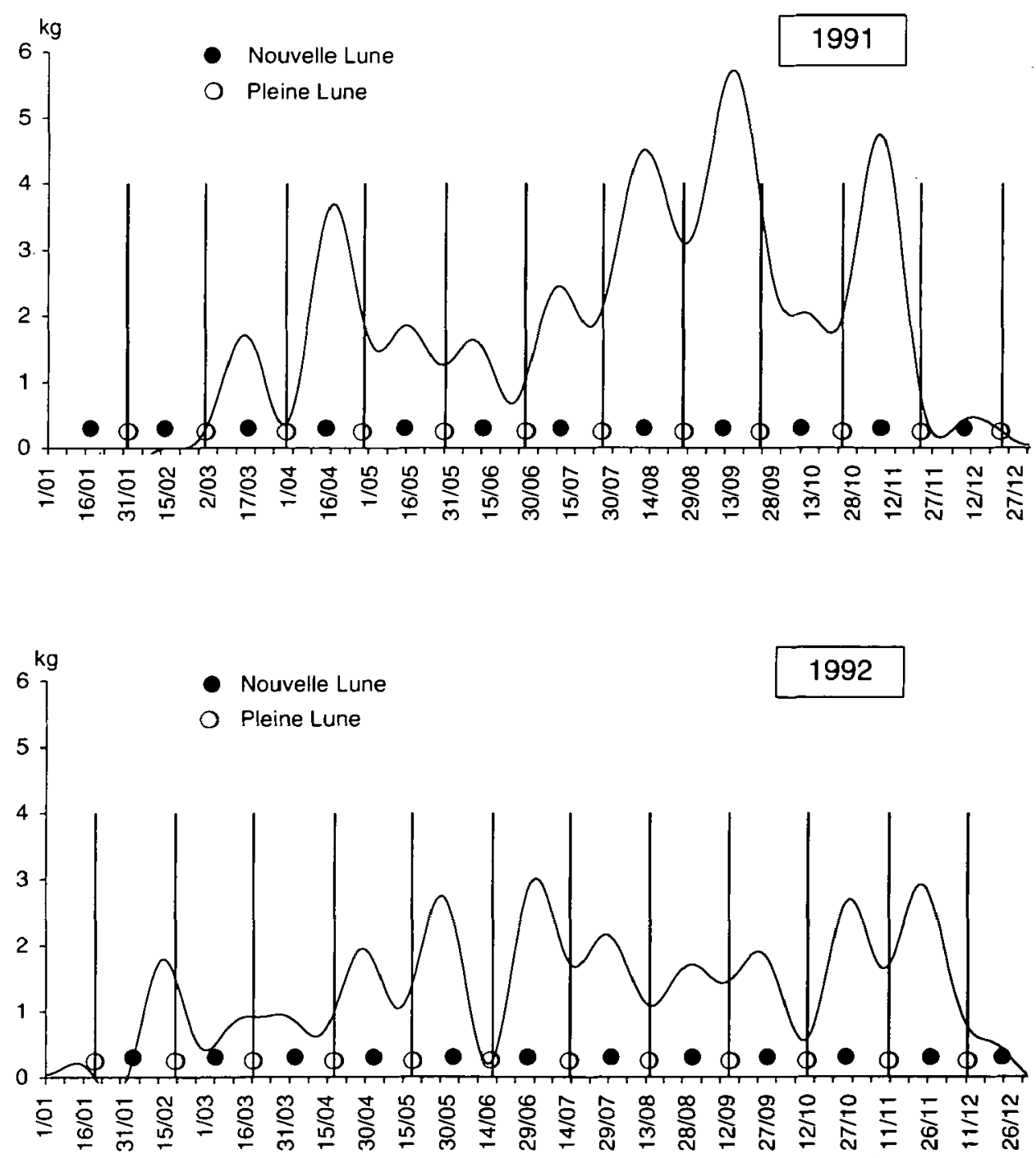

Figure 4 : Evolution lissée par un filtre passe-bas de TCHEBYCHEV (basé sur 28 jours) des captures d'anguille (Anguilla anguilla L.) par unité d'effort (Lac de Grand-Lieu, Loire-Atlantique) et projection du calendrier lunaire.

Figure 4 : Smooth evolution with lowpass filter of TCHEBYCHEV (based on 28 days) of catch per unit effort for eel (Anguilla anguilla L.) (Lake of Grand-Lieu, Loire-Atlantique) and projection of lunar calendar. 


\section{Comparaison du cycle lunaire et des captures}

La Figure 4 montre conjointement les variations de captures d'après le lissage de TCHEBYCHEV et les jours de pleine lune. Cette représentation graphique simple suffit à montrer la relation entre les phénomènes lunaires et les captures d'anguilles. Tout se passe comme si la pleine lune induisait un ralentissement des captures d'anguilles. En effet, au moment des pleines lunes, la courbe des captures s'infléchit nettement vers des valeurs moins fortes. Cet affaiblissement des captures est visible pour la totalité des 25 mois lunaires observés en 1991 et 1992.

\section{DISCUSSION}

Selon les études réalisées sur les déplacements des anguilles d'avalaison, deux hypothèses s'opposent pour expliquer le caractère inhibiteur de la lune. Certains auteurs mettent en avant le caractère lucifuge de l'anguille, ils expliquent ainsi que l'énergie lumineuse réfléchie par la lune et diffusée dans la masse d'eau induit chez ce poisson un état statique (LOWE, 1952 ; BOHUN et al., 1966 ; VAN-VEEN et al., 1976 et 1981 ; TODD, 1981 ; SLOANE, 1984 ; HARALDSTAD et al., 1985 ; VOLLESTAD et al., 1986 ; RUSSELLPOOLE et al., 1989). D'autres auteurs estiment que la luminosité n'est pas un facteur expliquant les déplacements d'anguilles d'avalaison (MEYER, 1938 ; KORRINGA, 1947 ; JENS, 1952 ; BOETIUS, 1967 ; EDEL, 1976 ; WESTIN et al., 1979). Ils s'appuient sur des expérimentations durant lesquelles les anguilles argentées étaient isolées de la lumière, mais répondaient toujours à un rythme lunaire. D'après KORRINGA (1947) et BOETIUS (1967), il s'agirait d'une adaptation de l'espèce pour minimiser la prédation.

Les deux hypothèses précédentes peuvent être retenues pour expliquer les variations de capture d'anguilles jaunes. II serait ainsi intéressant de tenter de lier les données de capture au facteur "luminosité nocturne", incluant à lui seul les phases lunaires, mais aussi les durées d'apparition de la lune durant la nuit, l'incidence des rayons lumineux d'origine lunaire, les passages nuageux, etc...

Nous venons de mettre en évidence l'existence d'un phénomène cyclique dans l'évolution des captures journalières d'anguilles sédentaires, ce phénomène est probablement dû à l'influence du cycle lunaire. Mais le comportement de l'anguille, et par conséquent sa capture, ne répond pas uniquement à ce phénomène. Bien d'autres paramètres sont connus pour être plus ou moins influents sur le comportement général des animaux aquatiques : la température, la pression atmosphérique, la pluviométrie, le taux d'insolation, le vent, pour ne citer que les facteurs météorologiques souvent à l'origine d'autres paramètres.

L'analyse de ces données est complexe, elle doit tenir compte du fait qu'il n'y a pas forcément de liaison directe entre les différents facteurs, ou encore que les liaisons ne sont pas linéaires. Un cheminement d'analyse calqué sur la "chaîne régression" définie dans le manuel de référence de la bibliothèque de programmes ADDAD (1989) est, semble-t-ii, capable de révéler les degrés de liaison entre les facteurs retenus et les données de captures.

A priori, la température semble avoir une influence sur les captures d'anguille. Cet effet est visible annuellement, les saisons d'hiver impliquent généralement un ralentissement des prises d'anguille notamment en dessous d'un seuil de température généralement situé aux alentours de $10^{\circ} \mathrm{C}$. Sur de plus courtes périodes, le phénomène déjà observé chez la civelle devra être vérifié pour ce stade biologique.

\section{REMERCIEMENTS}

Notre reconnaissance s'adresse tout particulièrement aux pêcheurs professionnels de Grand-Lieu pour l'accueil et l'aide qu'ils nous ont offerts.

Cette étude a bénéficié d'une convention CEMAGREF / Ministère de l'Environnement. 


\section{BIBLIOGRAPHIE}

ADAM G., ELIE P., 1993. Etude de la faune ichthyologique et de l'exploitation halieutique professionnelle du lac de Grand-Lieu, Loire-Atlantique. Premières descriptions et analyses. CEMAGREF, Bordeaux. 128 p.

BILDSTEIN P., 1990. Fonction et transfert des filtres électriques. Revue Technique de l'Ingénieur, E3, 110.5, 10-13.

BOETIUS J., 1967. Experimental indication of lunar activity in European silver eel Anguilla anguilla (L.). Meddelelser fra Danmarks Fiskeri, 6.

BOHUN S. et WINN H.E., 1966. Locomotion activity of the American eel (Anguilla rostrata). Chesapeake Science, 7, 137-147.

CREUTZBERG F., 1961. On the orientation of the migrating elvers ( $A$. vulgaris) in a tidal area. Neth. Jnl. Sea Res. 1 (3), 257-338.

DAHL J., 1983. Some observations on the ascent of young eels at the Tange Power dam, River Gudena. Communication Inland Fisheries Laboratory. Silkeborg, Danmark. $11 \mathrm{p}$.

DEELDER C.L., 1960. On the behaviour of elver (A. vulgaris) migrating from the sea into fresh-water. J. Cons. Perm. Int. Explor. Mer, 24 (1), 135-146.

DEELDER C.L., 1970. Synopsis of biological data on the eel Anguilla anguilla (Linnaeus) 1758. FAO Fish. Synops. $80 \mathrm{p}$.

EDEL R.K., 1976. Activity rythms of maturing American eels (Anguilla rostrata). Marine Biology, 36, 283-289.

ELIE P., 1979. Contribution à l'étude des montées de civelles d'Anguilla anguilla L. dans l'estuaire de la Loire : pêche, écophysiologie et élevage. Thèse de biologie animale. Rennes. $383 \mathrm{p}$.

ELIE P., ROCHARD E., 1994. Migration des civelles d'anguilles, (Anguilla anguilla L.), dans les estuaires, modalités du phénomène et caractéristiques des individus. Bull. Fr. Pêche Piscic., 335.

FINIGER R., 1976. Contribution à l'étude biologique et écologique des civelles (Anguilla anguilla Linné 1758) lors de leur pénétration dans un étang méditerranéen. 1Recrutement et biométrie au cours d'un cycle annuel. Vie-Milieu, 26 (1), 123-144.

GRAMAGLIA A., 1993. Traitement d'un signal biologique et ébauche d'un modèle de migration des poissons amphihalins. C.U.S.T., Institut des Sciences de l'Ingénieur. 39 p.

HARALDSTAD O., VOLLESTAD L.A., JONSSON B., 1985. Descent of European silver eels, Anguilla anguilla L., in a Norwegian watercourse. J. Fish. Biol., 6, 37-41.

JELLYMAN D.J., RYAN C.M., 1983. Seasonal migration of elvers (Anguilla spp.) into lake Pounui, New-Zealand, 1974-1978, N.Z. J. Mar. Freshwat. Res., 17, 1-15.

JENS G., 1952-53. Uber den lunaren rhythmus der blankaalwanderung. Arch. Fisckwin., $94,110$.

KORRINGA P., 1947. Relation between the moon and the periodicity in the breeding of marine animals. Ecol. Monogr., 17, 347-381.

LOWE R.H., 1952. The influence of light and other factors on the seaward migration of the silver eel (Anguilla anguilla L.). J. Anim. Ecol., 21. 275-309.

MEYER P.F., 1938. Die beeinflunung des blankaalfanges an der rugenkuste durch meteorologische und kosmische faktoren. $Z$. Fisch.

RUSSELL-POOLE W., REYNOLDS J.D., 1989. Observations on the silver eel runs of the burrishoole river system, Ireland, 1959 to 1988. EIFAC Working Party on Eel. 15 p. 
SLOANE R.D., 1984. Preliminary observation of migrating adult freshwater eels (Anguilla australis australis, Richardson) in Tasmania. Aust. J. Mar. Freshwat. Res., 35, 471-476.

TESCH F.W., 1977. The eel : Biology and management of anguillid eels. Translated from german by GREENWOOD, Chapman et Hall / Wiley and Sons, New-York. 435 p.

TODD P.R., 1981. Timing and periodicity of migrating New Zealand freshwater eels (Anguilla spp.). N.Z. J. Mar. Freshwat. Res., 15, 225-235.

VAN-VEEN T., HARTWIG H.G., MULLER K., 1976. Light dependent $n$, activity and photonegative behaviour in the eel (Anguilla anguilla L.). J. Comp. Physiol., 111, 209-219.

VAN-VEEN T., ANDERSON M., NILSSON D.E., 1981. Diel rythm in the elver Anguilla anguilla testing a computerized system for recording biorythms. Oikos, 37, 69-72.

VOLLESTAD L.A., JONSSON B., HVIDSTEN A.A., NAESTE T.F., HARALDSTAD O., RUUD-HANSEN J., 1986. Environmental factors regulating the seaward migration of European silver eels (Anguilla anguilla). Can. J. Fish. Aquat. Sci., 43, 1909-1916.

WESTIN L., NYMAN L., 1979. Activity, orientation, and migration of Baltic eel (Anguilla anguilla L.). Rapp. P.-V. Réun. ClEM, 174, 115-123. 